\title{
Сорбционные характеристики сорбентов для твердофазной экстракции фенольных соединений из экстрактов лекарственных растений
}

\author{
Шилько Е.А., Милевская В.В., Темердашев 3.А., Киселева Н.В. \\ ФГБОУ ВО «Кубанский государственный университет», Краснодар
}

Поступила в редакцию 11.02.2019 г.

DOI: https://doi.org/10.17308/sorpchrom.2019.19/733

В основе исследований настоящей работы находится твердофазное извлечение фенольных компонентов экстрактов лекарственного растительного сырья различными типами сорбционных материалов. В качестве сорбционных материалов использовали октадецилсиликагель (Strata C18-E), сополимер стирола и дивинилбензола, химически модифицированный N-винилпирролидоном, (Strata X), а также сополимер дивинилбензола и N-винилпирролидона (Oasis HLB). Для каждого из сорбентов получены сорбционные характеристики по отношению к целевым соединениям (объем до проскока, равновесный объем, объем удерживания и др.), а также десорбционные параметры (коэффициент концентрирования и степень извлечения). Показано, что сорбенты на основе октадецилсиликагеля достаточно эффективны для извлечения группы флавоноидов и их гликозидов, а полимерные материалы - одновременно и флавоноидов, и фенольных кислот. Степени извлечения фенольных кислот и флавоноидов при коэффициенте концентрирования 5 на сорбенте Strata C18-E составили 2740\% и $79-105 \%$ соответственно. С применением сорбента Strata X также достигается концентрирование в 5 раз при степенях извлечения 106-107\% (для фенольных кислот) и 99-122\% (для флавоноидов). Ha сорбенте Oasis HLB возможно концентрирование в 20 раз фенольных кислот при их степенях извлечения 74-96\% и в 32 раза для флавоноидов при степенях извлечения в диапазоне 56-91\%.

Ключевые слова: твердофазная экстракция, сорбция, десорбция, фенольные кислоты, флавоноиды, лекарственное растительное сырье, высокоэффективная жидкостная хроматография, Strata C18-E, Strata X, Oasis HLB

\section{Sorption characteristics of sorbents for solid-phase extraction of phenolic compounds from extracts of medicinal plants}

\author{
Shil'ko E.A., Milevskaya V.V., Temerdashev Z.A., Kiseleva N.V.
}

Kuban State University, Krasnodar

Extraction of different compounds from plant materials and their concentration is an actual task, which is solved by introducing a solid phase extraction (SFE) stage into the analytical cycle. Currently, there is a large variety of sorbents that allow extracting a wide range of compounds from various matrixes, for instance, biologically active components from aqueous and aqueous-alcoholic extracts of medicinal raw material. It is possible to assess the possibility and expediency of applying any sorption material for SPE of target groups of compounds by obtaining and further comparing their sorption characteristics. Based on this, the goal of our work was to obtain and study the main sorption characteristics of a number of concentrating sorbents of various chemical structures for the SPE of phytocomponents from medicinal plant extracts. As sorption materials, we used sorbents based on octadecylsilicagel (Strata C18-E), styrene and divinylbenzene co- 
polymer, chemically modified N-vinylpyrrolidone, (Strata X), and also divinylbenzene and Nvinylpyrrolidone copolymer (Oasis HLB).

For experiment we obtained water extracts of Hypericum, which were acidified with $1 \% \mathrm{HCl}$ solution to $\mathrm{pH}=2$ to obtain the output dynamic sorption curves. At this step extracts were passed through cartridges at a rate of $1 \mathrm{~cm}^{3} / \mathrm{min}$, taking the corresponding fractions into vials. Next, the sorbent was dried in a stream of nitrogen and the desorption curves of the phenolic components were obtained. The content of the target compounds in the eluates was controlled by HPLC-DAD system.

The main sorption characteristics were obtained for all target compounds («the breakthrough volume», the equilibrium volume, the retention volume, etc.), as well as the desorption parameters (concentration ratio and percentage of recovery). «The breakthrough volumes» of phenolic acids using the Strata X and Oasis HLB sorbents were 5 and $9 \mathrm{~cm}^{3}$, respectively, in contrast to the Strata C18-E sorbent («the breakthrough volumes» were not more than $0.5 \mathrm{~cm}^{3}$ ). For SPE of flavonoids on polymer materials, «the breakthrough volumes» increase several times as compared with the Strata C18 sorbent (not more than $1 \mathrm{~cm}^{3}$ ) and reaches 6 and $19 \mathrm{~cm}^{3}$ for Strata X and Oasis HLB, respectively. The Strata C18-E recoveries of phenolic acids and flavonoids were $27-40 \%$ and $79-105 \%$ respectively when the concentration ratio was 5 . Applying the Strata $\mathrm{X}$ sorbent, the concentration is also achieved 5 times with recoveries of $106-107 \%$ (for phenolic acids) and $99-122 \%$ (for flavonoids). Applying the Oasis HLB sorbent, it is possible to concentrate phenolic acids by 20 times with their recoveries of $74-96 \%$ and flavonoids by 32 times with recoveries in the range of 56$91 \%$.

Thus, it was shown that sorbents based on octadecylsilagel are rather effective for extracting a group of flavonoids and their glycosides, and polymeric materials are both flavonoids and phenolic acids at the same time, moreover the maximum concentration coefficients were observed applying the Oasis HLB.

Keywords: solid phase extraction, sorption, desorption, phenolic acids, flavonoids, medicinal raw material, HPLC-DAD, Strata C18-E, Strata X, Oasis HLB

\section{Введение}

Извлечение соединений различного состава из растительных материалов и их концентрирование - актуальная задача, которую решают путем введения стадии твердофазной экстракции в аналитический цикл. Эффективность твердофазной экстракции как способа концентрирования веществ определяется физико-химическими процессами между материалом сорбента и целевыми соединениями, из которых наиболее часто реализуются ван-дер-ваальсовы, водородные, диполь-дипольные и катионно-анионные взаимодействия. В настоящее время известно большое разнообразие сорбентов, позволяющих извлекать широкий спектр соединений из различных сред, в том числе и биологически активные компоненты (фенолкарбоновые и коричные кислоты, флавоноиды и их гликозиды различного состава, а также полифенольные соединения) из водных и водно-спиртовых экстрактов лекарственных растений (ЛР). Наиболее часто для извлечения и концентрирования данных групп соединений используют октадецилсиликагель [1-6] и полимерные материалы [3, 7-12]. Сорбенты на основе октадецилсиликагеля используют, в основном, для очистки экстрактов ЛР без концентрирования целевых соединений $[2,13,14]$. Полимерные материалы, в том числе сорбенты с гидрофильно-липофильным балансом, в отличие от сорбентов с привитыми октадецильными группами, используют также и для концентрирования фенолкарбоновых кислот и флавоноидов [7, 9, 10, 15].

Оценить возможность и целесообразность применения того или иного сорбционного материала для ТФЭ целевых групп соединений возможно путем получения и дальнейшего сравнения их сорбционных характеристик. Одной из ключевых сорбционных характеристик является «объем до проскока», который может определяться графически исходя из выходной динамической кривой сорбции на уровне 5-10\% от исходной концентрации соединения в экстракте. В идеальном случае, выходная динамическая кривая сорбции имеет билогарифмическую сигмоидальную форму. Кроме «объема до проскока» графически можно получить такие характеристики, как объем удерживания и равновесный объем [16]. Важными сорбционными характери- 
стиками при подборе сорбента для ТФЭ фенольных соединений являются десорбционные параметры - коэффициент концентрирования и степень извлечения. Для десорбции фенолкарбоновых и коричных кислот, флавоноидов и их гликозидов из экстрактов ЛР используют спирты, в частности, метанол [7, 17, 18].

В большинстве работ используют один тип сорбента для извлечения фенольных компонентов из экстрактов лекарственного растительного сырья $[2,7,9,12]$, а сравнение эффективности сорбентов различного состава по отношению к одному виду ЛР практически не проводилось.

Целью нашей работы являлось получение и изучение основных сорбционных характеристик ряда концентрирующих сорбентов различной структуры для твердофазного извлечения фитокомпонентов из экстрактов ЛР.

\section{Эксперимент}

Объектом исследования выбрана трава зверобоя (Hypericum perforatum L.) («Травы Кавказа», Краснодарский край, Горячий Ключ), измельченная до размеров частиц 0.5-1 мм.

Растворители, реагенты и стандартные образцы. Для получения деионизованной воды с удельным сопротивлением 18.2 МОм·см (при $25^{\circ} \mathrm{C}$ ) была применена установка Milli-Q-UV (Millipore, Франция). Экспериментальные исследования проводили с использованием ацетонитрила (HPLC-S, «Biosolve BV», Netherland), муравьиной кислоты (85\%, «ЛенРеактив», Россия), соляной кислоты (ч, «Реахим», Россия) и метанола (хч, «Вектон», Россия). Идентификацию аналитов проводили с применением стандартных образцов 3,4-дигидроксибензойной, неохлорогеновой и хлорогеновой кислот, (-)-эпикатехина, рутина, гиперозида, изокверцитрина, кверцитрина (Sigma-Aldrich, Германия).

Оборудование. Контроль $\mathrm{pH}$ экстрактов проводили рН-метр-иономером «Эксперт-001» (ООО «Эконикс-Эксперт», Россия). Хроматографическое определение компонентов осуществляли с помощью хроматографа «LC 20 Prominence» (Shimadzu, Япония) (дегазатор DGU-20A5, насос LC20AD, автоматический дозатор SIL$20 \mathrm{~A}$, термостат колонок СТО-20AC, спектрофотометрический детектор на основе диодной матрицы SPD-M20A). Для экстракции целевых компонентов из зверобоя использовали микроволновую установку ETHOS EX («Milestone», Италия).

Сорбционные материалы. В качестве сорбционных материалов были использованы патроны: Strata C18-E (Phenomenex, США), массой 100 мг с размером частиц 55 мкм и диаметром пор 7 нм; Strata X (Phenomenex, США), массой 100 мг с размером частиц 33 мкм и диаметром пор сорбента 8,5 нм; Oasis HLB (Waters, CША), мacсой 100 мг с размером частиц 30 мкм и диаметром пор 8 нм. Кондиционирование сорбентов осуществляли путем последовательного промывания $1 \mathrm{~cm}^{3}$ деионизованной воды, $1 \mathrm{~cm}^{3}$ метанола и $5 \mathrm{~cm}^{3}$ деионизованной воды [19].

Получение экстракта из растительного материала. Экстракцию фенольных соединений из ЛРС проводили под воздействием микроволнового излучения по методике, изложенной в работе [20], но в качестве экстрагента использовали деионизованную воду.

Изучение сорбционных характеристик. Для получения выходных динамических кривых сорбции водные экстракты зверобоя подкисляли 1\%-ным раствором $\mathrm{HCl}$ до $\mathrm{pH}=2$ [21], затем полученный экстракт пропускали через патроны со скоростью $1 \mathrm{~cm}^{3} /$ мин, отбирая соответствующие фракции в виалы. Сорбент сушили в токе азота при помощи генератора азота LCMS30-1 (Domnick Hunter, CША). Затем при заданной скорости потока метанола, получали кривые десорбции, используя насос 
LC20AD (Shimadzu, Япония). Содержание определяемых компонентов в элюатах контролировали с помощью метода ВЭЖХ-ДМД. «Объемы до проскока», объем удерживания, равновесный объем и число теоретических тарелок сорбентов рассчитывали графически и по формулам [16].

Хроматографическое определение фенолкарбоновых кислот и флавоноидов в экстрактах зверобоя. Определение фенолкарбоновых кислот и флавоноидов проводили по методике [20]. Разделение компонентов проводили на колонке Luna C18 100A, 250×2.0 мм, 5 мкм (Phenomenex, США) с предколонкой С18 4×2.0 мм, 5 мкм (Phenomenex, США), а полученные данные обрабатывали в программной среде LCMS Solution (Shimadzu, Япония).

\section{Обсуждение результатов}

Для построения выходных динамических кривых сорбции необходимо было исключить преждевременное элюирование таких гидрофильных соединений, как фенолкарбоновые кислоты с материала используемых сорбентов во время сорбции [22]. Оптимизируя условия сорбции компонентов, учитывали влияние $\mathrm{pH}$, физикохимических свойств определяемых соединений, природу матрицы анализируемого объекта, а также скорость пропускания экстракта через патрон. Фенольные соединения в водных экстрактах ЛР переводили в неионизированную форму $(\mathrm{pH}=2) 1 \%$-ой соляной кислотой для повышения сорбционного сродства.

Сорбционные характеристики химически модифицированного силикагеля Strata C18-Е. Для твердофазной экстракции фенольных соединений, как правило, используют сорбенты на основе силикагеля, модифицированного различными функциональными группами, придающими сорбентам определенные свойства (неполярные, полярные или смешанного характера) [5, 8]. Для сорбента Strata C18-E были получены выходные динамические кривые сорбции сигмоидальной формы для фенолкарбоновых кислот, флавоноидов и их гликозидов. Концентрирование фенольных соединений на октадецилсилане затруднено, о чем свидетельствует резкий скачок на начальном участке динамической кривой сорбции фенолкарбоновых кислот (рис. 1). Данный факт подтверждается также рассчитанными «объемами до проскока», которые составили $0.1,0.3$ и $0.4 \mathrm{~cm}^{3}$ для 3,4-дигидроксибензойной, неохлорогеновой и хлорогеновой кислот соответственно (табл. 1). Рассчитанные «объемы до проскока» (не более $1 \mathrm{~cm}^{3}$ ) и сигмоидальные формы кривых сорбции флавоноидов и их гликозидов также свидетельствуют о невысоких коэффициентах их концентрирования (табл. 1). Внутри каждой группы изучаемых соединений формы выходных динамических кривых сорбции близки (рис. 1 и 2), что можно интерпретировать схожестью физико-химических свойств компонентов, и обусловлены, по-видимому, наличием родственных функциональных групп. Как следует из таблицы 1, насыщение сорбента с привитыми октадецильными группами фенолкарбоновыми кислотами достигается после пропускания $5 \mathrm{~cm}^{3}$ водного экстракта ЛР, а флавоноидами и их гликозидами - $13 \mathrm{~cm}^{3}$. Полученные значения свидетельствуют о малой емкости данного типа сорбента по отношению к фенолкарбоновым кислотам, а для группы флавоноидов возможно получение невысоких коэффициентов концентрирования. По полученным сорбционным характеристикам рассчитывали число теоретических тарелок (ЧТТ) для октадецилсиликагеля по отношению к каждому анализируемому соединению. Высокие значения ЧТТ можно соотнести с большей эффективностью применения данного типа сорбента для концентрирования только флавоноидов. 


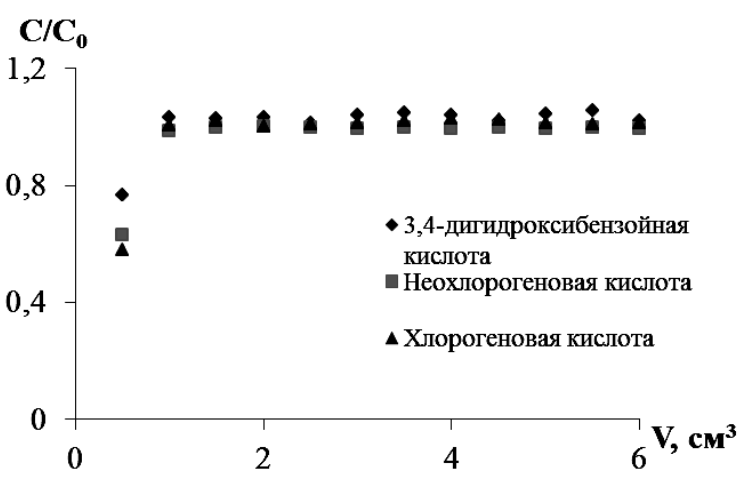

Рис. 1. Выходные динамические кривые сорбции фенолкарбоновых кислот на сорбенте Strata C18-E

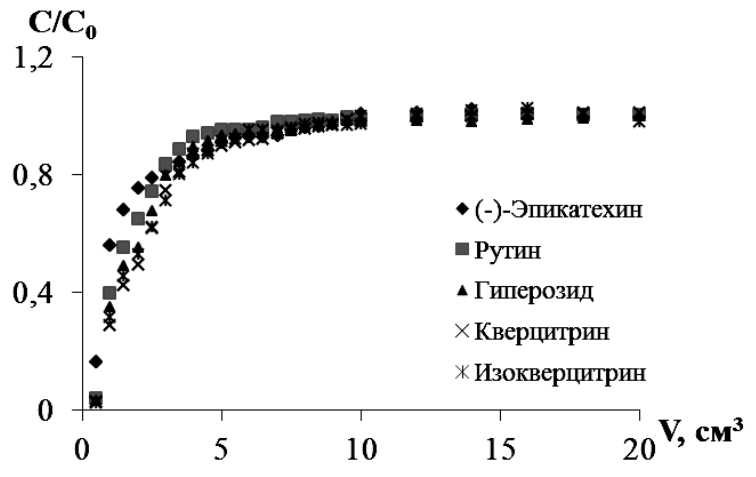

Рис. 2. Выходные динамические кривые сорбции флавоноидов и их гликозидов на сорбенте Strata C18-E

Таблица 1. Основные сорбционные параметры сорбента Strata C18-E по отношению к некоторым компонентам зверобоя*

\begin{tabular}{|c|c|c|c|c|c|c|c|c|c|c|}
\hline Сорбент & Соединение & $\begin{array}{l}\mathrm{V}_{\mathrm{R}_{3}} \\
\mathrm{CM}^{3}\end{array}$ & $\begin{array}{l}\mathrm{V}_{\mathrm{B}_{3}} \\
\mathrm{CM}_{3}\end{array}$ & $\begin{array}{l}\sigma_{\mathrm{V}_{1}} \\
\mathrm{~cm}^{3}\end{array}$ & $\begin{array}{l}\sigma_{\mathrm{V}}^{*}, \\
\mathrm{~cm}^{3}\end{array}$ & $\begin{array}{l}\mathrm{V}_{\mathrm{E}_{3}} \\
\mathrm{Cm}^{3}\end{array}$ & $\mathrm{~N}$ & $\mathrm{~S}_{\mathrm{r}}$ & K & $\mathrm{R}, \%$ \\
\hline \multirow{8}{*}{$\begin{array}{l}\text { Strata } \\
\text { C18-E }\end{array}$} & $\begin{array}{c}3,4- \\
\text { дигидроксибензойная } \\
\text { кислота }\end{array}$ & 0.9 & 0.1 & 0.4 & 0.5 & 2 & 3 & $\leq 0.5$ & 5 & \multirow{3}{*}{$27-40$} \\
\hline & $\begin{array}{c}\text { Неохлорогеновая } \\
\text { кислота }\end{array}$ & 1.3 & 0.3 & 0.5 & 0.8 & 3 & 4 & .5 & 5 & \\
\hline & Хлорогеновая кислота & 2.2 & 0.4 & 0.9 & 1.4 & 5 & 3.5 & $\leq 0.3$ & 5 & \\
\hline & (-)-эпикатехин & 2.5 & 0.4 & 1 & 1 & 5 & 4 & $\leq 0.2$ & 5 & \multirow{5}{*}{$79-105$} \\
\hline & Рутин & 4 & 0.7 & 0.6 & 0.3 & 11 & 38 & $\leq 0.2$ & 5 & \\
\hline & Гиперозид & 5 & 0.6 & 0.6 & 0.5 & 13 & 61 & $\leq 0.3$ & 5 & \\
\hline & Изокверцитрин & 5 & 0.6 & 0.6 & 0.5 & 13 & 61 & $\leq 0.2$ & 5 & \\
\hline & Кверцитрин & 5 & 0.6 & 0.6 & 0.5 & 13 & 61 & $\leq 0.3$ & 5 & \\
\hline
\end{tabular}

* $\mathrm{V}_{\mathrm{R}}$ - объем удерживания; $\mathrm{V}_{\mathrm{B}}-$ «объем до проскока»; $\mathrm{V}_{\mathrm{E}}-$ равновесный объем; $\sigma_{\mathrm{V}}$ и $\sigma_{\mathrm{V}}{ }^{*}-$ стандартные отклонения на выходной динамической кривой сорбции, $\mathrm{N}$ - число теоретических тарелок; К - коэффициент концентрирования; $\mathrm{R}$ - степень извлечения.

Сорбционные характеристики полимерных сорбентов Strata X и Oasis HLB. Для сорбции фенольных соединений наиболее широко используются макропористые [23] полимерные сорбенты, представляющие собой сополимеры стирола и дивинилбензола, и за счет наличия гидрофобной полимерной структуры они более эффективны для концентрирования соединений с большим количеством бензольных колец. Одними из преимуществ этих полимерных сорбентов являются возможность работы с ними в широком диапазоне $\mathrm{pH}$ [24] и отсутствие силанольных групп, характерных для октадецилсилана, что снижает эффект необратимой сорбции аналитов. При сорбции фенольных соединений на полимерных сорбентах возможны диполь-дипольные, гидрофобные, $\pi-\pi$ и межмолекулярные взаимодействия между структурой аналита и материалом $[19,25]$. Полимерные материалы могут также модифицироваться путем присоединения различных полярных функциональных групп (например, ацетил-, бензоил- и гидроксометил-групп), обеспечивающих лучший массоперенос и взаимодействие аналитов с функциональными группами [23]. Получаемые химически модифицированные полимерные смолы обладают большей гидрофильностью и показывают высокие степени извлечения аналитов за счет увеличения полярности поверхности сорбента.

На характер удерживания фенольных соединений, по-видимому, может влиять различный механизм полимеризации сорбентов. Сорбент Oasis HLB химически 
представляет собой макропористый продукт полимеризации липофильного дивинилбензола и гидрофильного N-винилпирролидона. Сорбент Strata X представляет собой сополимер стирола и дивинилбензола, поверхностно-модифицированный Nвинилпирролидоном [26]. Исходя из этого и анализируя данные по сорбции некоторых фенольных соединений (табл. 2) можно предположить, что сорбция компонентов на представленных полимерных сорбентах протекает по различным механизмам.

Таблица 2. Основные сорбционные параметры полимерных сорбентов Strata X и Oasis HLB по отношению к некоторым компонентам зверобоя*

\begin{tabular}{|c|c|c|c|c|c|c|c|c|c|c|}
\hline Сорбент & Соединение & $\begin{array}{l}\mathrm{V}_{\mathrm{R}_{3}} \\
\mathrm{CM}^{3}\end{array}$ & $\begin{array}{l}\mathrm{V}_{\mathrm{B}_{3}} \\
\mathrm{~cm}^{3}\end{array}$ & $\begin{array}{l}\sigma_{\mathrm{V}} \\
\mathrm{cm}^{3}\end{array}$ & $\begin{array}{l}\sigma_{\mathrm{V}^{*}} \\
\mathrm{~cm}^{3}\end{array}$ & $\begin{array}{l}\mathrm{V}_{\mathrm{E}} \\
\mathrm{cm}^{3}\end{array}$ & $\mathrm{~N}$ & $\mathrm{~S}_{\mathrm{r}}$ & $\mathrm{K}$ & $\mathrm{R}, \%$ \\
\hline \multirow{8}{*}{ Strata X } & $\begin{array}{c}3,4- \\
\text { дигидроксибензойная } \\
\text { кислота }\end{array}$ & 6 & 5 & 1 & 1 & 8 & 30 & $\leq 0.3$ & 5 & \multirow{3}{*}{$\begin{array}{l}106- \\
107\end{array}$} \\
\hline & $\begin{array}{c}\text { Неохлорогеновая ки- } \\
\text { слота }\end{array}$ & 6 & 4 & 1 & 1 & 7 & 30 & $\leq 0.4$ & 5 & \\
\hline & $\begin{array}{c}\text { Хлорогеновая кисло- } \\
\text { та }\end{array}$ & 8 & 5 & 1 & 1 & 10 & 56 & $\leq 0.6$ & 5 & \\
\hline & (-)-эпикатехин & 9 & 6 & 2 & 1 & 11 & 16 & $\leq 0.3$ & 5 & \multirow{5}{*}{$\begin{array}{l}99- \\
122\end{array}$} \\
\hline & Рутин & 13 & 3 & 5 & 3 & 18 & 4 & $\leq 0.2$ & 5 & \\
\hline & Гиперозид & 14 & 6 & 4 & 2 & 23 & 9 & $\leq 0.2$ & 5 & \\
\hline & Изокверцитрин & 14 & 6 & 4 & 3 & 23 & 9 & $\leq 0.2$ & 5 & \\
\hline & Кверцитрин & 16 & 6 & 5 & 7 & 30 & 7 & $\leq 0.2$ & 5 & \\
\hline \multirow{8}{*}{$\begin{array}{l}\text { Oasis } \\
\text { HLB }\end{array}$} & $\begin{array}{c}3,4- \\
\text { дигидроксибензойная } \\
\text { кислота }\end{array}$ & 13 & 9 & 2 & 2 & 17 & 36 & $\leq 0.1$ & 20 & \multirow{3}{*}{$\begin{array}{l}74- \\
96\end{array}$} \\
\hline & $\begin{array}{c}\text { Неохлорогеновая ки- } \\
\text { слота }\end{array}$ & 11 & 9 & 1.3 & 2.3 & 16 & 63 & $\leq 0.3$ & 20 & \\
\hline & $\begin{array}{c}\text { Хлорогеновая кисло- } \\
\text { та }\end{array}$ & 15 & 7 & 4 & 5 & 25 & 10 & $\leq 0.1$ & 20 & \\
\hline & (-)-эпикатехин & 18.5 & 12.5 & 3 & 3.8 & 26 & 32 & $\leq 0.1$ & 20 & \multirow{5}{*}{$\begin{array}{l}56- \\
91\end{array}$} \\
\hline & Рутин & 26 & 17 & 4.8 & 2 & 30 & 24 & $\leq 0.1$ & 32 & \\
\hline & Гиперозид & 30 & 18 & 6.3 & 4.8 & 40 & 18 & $\leq 0.1$ & 32 & \\
\hline & Изокверцитрин & 31 & 19 & 5.8 & 5 & 41 & 23 & $\leq 0.1$ & 32 & \\
\hline & Кверцитрин & 35 & 19 & 8.3 & 6.8 & 49 & 14 & $\leq 0.1$ & 32 & \\
\hline
\end{tabular}

* $\mathrm{V}_{\mathrm{R}}$ - объем удерживания; $\mathrm{V}_{\mathrm{B}}$ - «объем до проскока»; $\mathrm{V}_{\mathrm{E}}-$ равновесный объем; $\sigma_{\mathrm{V}}$ и $\sigma_{\mathrm{V}}{ }^{*}-$ стандартные отклонения на выходной динамической кривой сорбции, $\mathrm{N}$ - число теоретических тарелок; /К - коэффициент концентрирования; $\mathrm{R}$ - степень извлечения.

Для флавоноидов и фенолкарбоновых кислот наблюдается сигмоидальная форма выходных динамических кривых сорбции на Strata X и Oasis HLB (рис. 3-6). Практически для всех исследуемых соединений на выходных динамических кривых сорбции наблюдается подъем кривой выше $\mathrm{C} / \mathrm{C}_{0}=1$, что может быть обусловлено одновременными процессами сорбции и концентрирования при пропускании водного экстракта растительного образца через сорбент. Рассчитанные «объемы до проскока» для фенолкарбоновых кислот на сорбенте Strata X составили 5 см$^{3}$, что позволяет концентрировать данные группы соединений в отличие от применения для этих целей сорбента Strata C18-Е. При использовании Oasis HLB «объем до проскока» фенолкарбоновых кислот увеличивается до $9 \mathrm{~cm}^{3}$, что свидетельствует о повышении коэффициента концентрирования изучаемых соединений $(K=20)$. При проведении твердофазного извлечения флавоноидов на полимерных материалах «объем до проскока» для данной группы соединений увеличивается в несколько раз по сравнению 
с сорбентом Strata C18 и достигает 6 и 19 cm $^{3}$ для Strata X и Oasis HLB соответственно, что также обеспечивает увеличение коэффициента концентрирования целевых соединений.

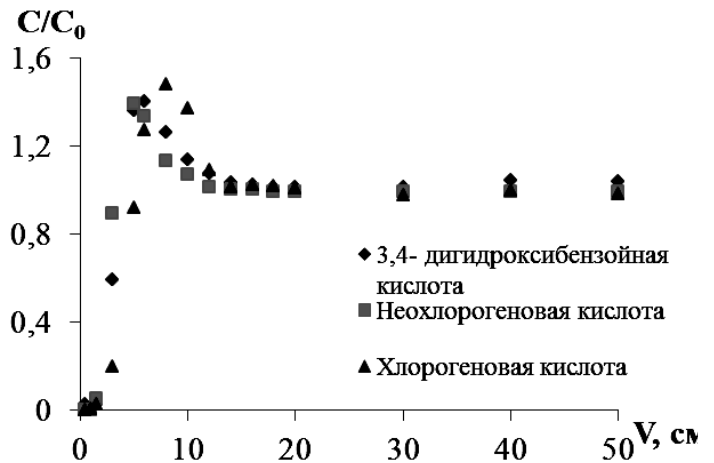

Рис. 3. Выходные динамические кривые сорбции фенолкарбоновых кислот на сорбенте Strata X

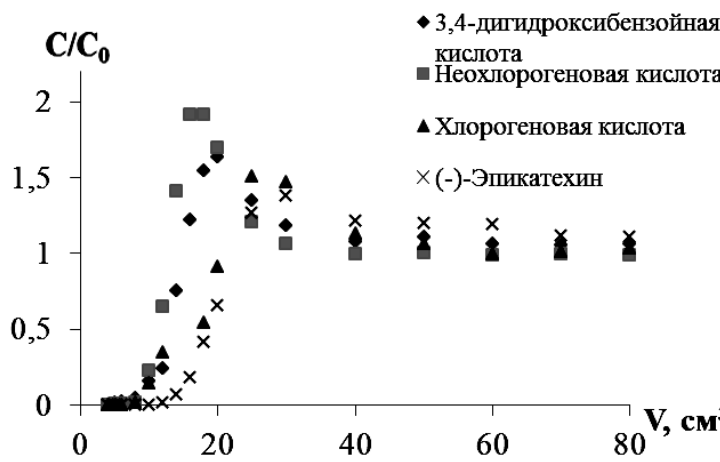

Рис. 5. Выходные динамические кривые сорбции фенолкарбоновых кислот на сорбенте Oasis HLB

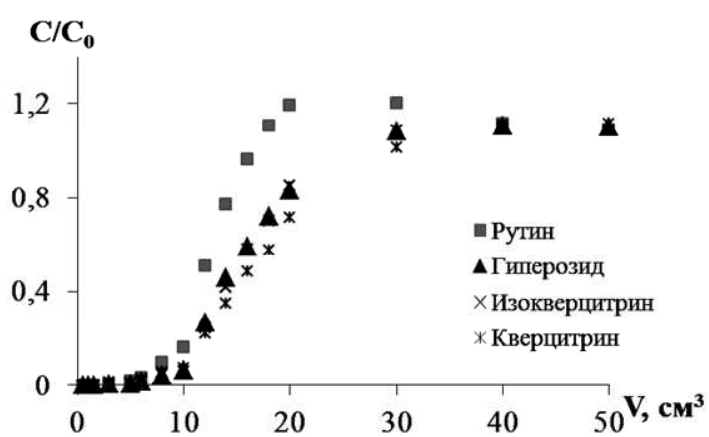

Рис. 4. Выходные динамические кривые сорбции флавоноидов и их гликозидов на сорбенте Strata X

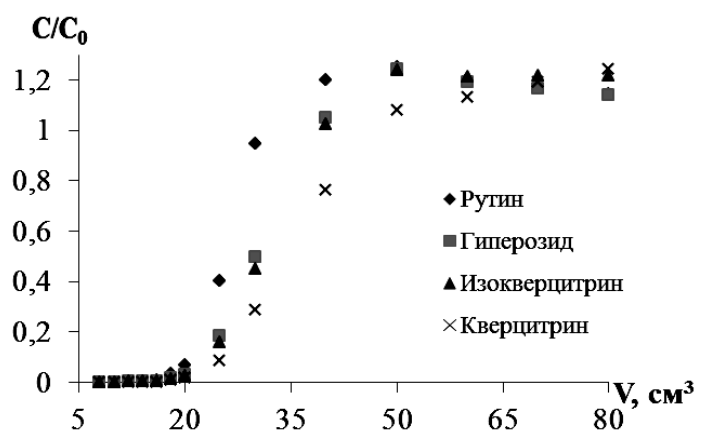

Рис. 6. Выходные динамические кривые сорбции флавоноидов и их гликозидов на сорбенте Oasis HLB

Значения основных характеристик, полученных из выходных динамических кривых сорбции фенольных соединений на полимерных сорбентах Strata X и Oasis HLB, представлены в табл. 2. Рассчитанные значения ЧТТ полимерных сорбентов по отношению к фенолкарбоновым кислотам позволяют говорить об их большей целесообразности применения для концентрирования данной группы соединений. Примечательно, что значения ЧТТ полимерных сорбентов по отношению к группе флавоноидов и их гликозидам значительно меньше данной характеристики, полученной для октадецилсиликагелевого сорбента, что говорит о меньшем сорбционном сродстве данных материалов и аналитов. Разница в ЧТТ для Oasis HLB и Strata X по отношению к группе флавоноидов обусловлена, по-видимому, гидрофильнолипофильной природой первого сорбента.

Изучение процессов десорбции фенольных соединений зверобоя. Кривые десорбции компонентов с сорбентов позволяют сделать вывод о возможной степени концентрирования соединений, предоставляя информацию о достаточном объеме растворителя для смыва компонентов с сорбента. Анализируя вид кривых десорбции целевых компонентов с сорбентов (рис. 7-9) можно отметить, что десорбция соединений протекает по-разному.

Исходя из кривой десорбции фенольных соединений метанолом, полученной на сорбенте Strata C18-E (рис. 7), можно заметить, что подавляющая часть компо- 
нентов десорбируется $0.2 \mathrm{~cm}^{3}$ метанола. При извлечении флавоноидов метанолом с поверхности Strata C18-Е удалось получить степень извлечения 79 - 105\% при концентрировании в 5 раз. Добиться высоких степеней извлечения фенолкарбоновых кислот, превышающих 40\%, на данном типе сорбента при концентрировании в 5 раз не удалось, вследствие достаточно быстрого достижения «проскока» данных соединений.

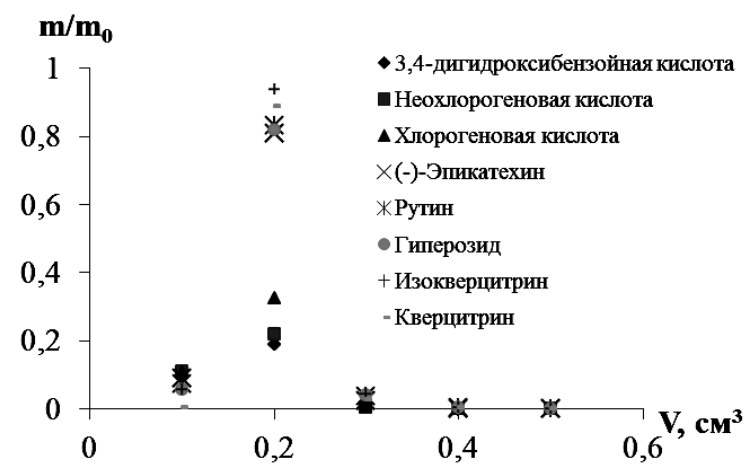

Рис. 7. Кривая десорбции фенольных соединений зверобоя метанолом с сорбента Strata C18-E.

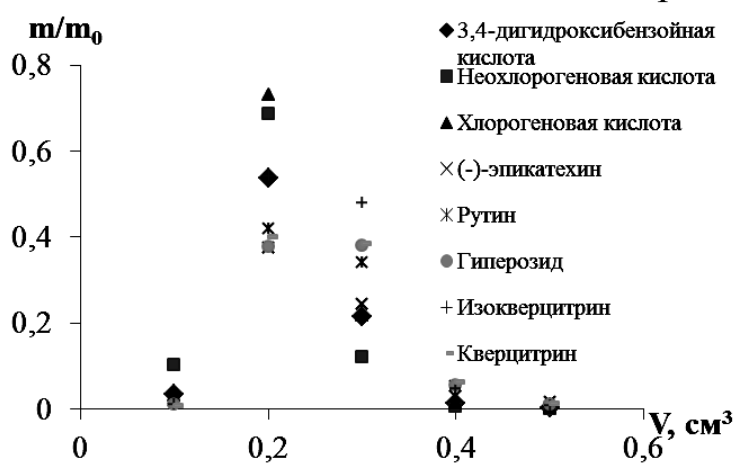

Рис. 9. Кривая десорбции фенольных соединений зверобоя метанолом с сорбента Oasis HLB.

В отличие от сорбента с привитыми октадецильными группами, подавляющая часть фенольных соединений десорбируется с полимерного Strata X 0.1-0.2 см ${ }^{3}$ метанола (рис. 8). При концентрировании в 5 раз степень извлечения фенолкарбоновых кислот из экстракта зверобоя составила 106-107\% и 99-112\% для группы флавоноидов и их гликозидов. При использовании альтернативного полимерного материала (Oasis HLB) объемы десорбции веществ составили 0.2-0.3 $\mathrm{cm}^{3}$ (рис. 9). С помощью данного сорбента удалось повысить коэффициент концентрирования фенольных кислот и флавоноидов до 20 и 32 соответственно благодаря увеличению «объемов до проскока» всех исследуемых соединений на данном материале. Однако при этом степень извлечения фенолкарбоновых кислот варьировалась от $74 \%$ до 96\%, а флавоноидов - от 56 до 91\% (табл. 2). Разброс степени извлечения соединений на сорбенте Oasis HLB, вероятно, может говорить о частично-необратимой адсорбции некоторых компонентов. Таким образом, на полимерном сорбенте Strata X достигаются высокие степени извлечения (более 99\%) обеих групп соединений при их концентрировании в 5 раз. Напротив, на сорбенте Oasis HLB коэффициенты концентрирования фенольных кислот и флавоноидов в 5 и 6 раз соответственно превышают таковые для Strata X, однако степени извлечения для некоторых индивидуальных соеди- 
нений неудовлетворительны при использовании сорбента с липофильногидрофильным балансом. Сорбент Strata C18-E обеспечивает пятикратное концентрирование флавоноидов при степени извлечения выше $79 \%$.

\section{Заключение}

В работе показана эффективность использования различных типов сорбционных материалов для целей извлечения и концентрирования фенольных соединений из водных экстрактов лекарственных растений. Установлено, что сорбенты на основе октадецилсиликагеля эффективны по отношению к группе флавоноидов, а полимерные сорбенты - по отношению к обеим группам соединений. Получены основные сорбционные параметры для каждого типа сорбента по отношению к целевым соединениям - «объем до проскока», объем удерживания и равновесный объем, рассчитаны число теоретических тарелок по отношению к каждому определяемому соединению, а также коэффициенты их концентрирования и степени извлечения. В результате показана перспективность использования полимерных сорбентов с гидрофильно-липофильным балансом для извлечения и концентрирования до 32 раз соединений фенольной природы из растительной матрицы.

Исследования проводили в рамках выполнения проекта Государственного Задания Минобрнауки РФ (№4.2612.2017/ПЧ) при финансовой поддержке РФФИ

(проект № 18-33-20009 мол_а_вед) с использованием научного оборудования ЦКП «Экологоаналитический цент» Кубанского госуниверситета, уникальный идентификатор RFMEFI59317X0008.

\section{Список литературы}

1. Zgorka G., Hajnos A. // Chromatographia. 2003. Vol. 57. No 1. pp. 77-80.

2. Kozyra M., Glowniak K. // Acta Soc. Bot. Pol. 2013. Vol. 82. No 4. pp. 325-329.

3. Zeng H., Liu Z., Zhao S., Shu Y. et al. // J. Sep. Sci. 2016. Vol. 39. No 19. pp. 3806-3817.

4. Glowniak K., Zgorka G., Kozyra M. // J. Chromatogr. A. 1996. Vol. 730. pp. 25-29.

5. Oniszczuk A., Podgorski R., Oniszczuk T., Zukiewicz-Sobczak W. et al. // Industrial Crops and Products. 2014. Vol. 61. pp. 377-381.

6. Skrzypczak-Pietraszek E., Pietraszek J. // J. Pharm. Biomed. Anal. 2012. Vol. 66. Pp 154161.

7. Stephen Inbaraj B., Lu H., Kao T.H., Chen B.H. // J. Pharm. Biomed. Anal. 2010. Vol. 51. pp. 549-556.

8. Irakli M.N., Samanidou V.F., Biliaderis C.G., Papadoyannis I.N. // J. Sep. Sci. 2012. Vol. 35. pp. 1603-1611.

9. Ziakova A., Brandsteterova E. // J. Liq. Chromatogr. Relat. Technol. 2002. Vol. 25. No 19. pp. 3017-3032.
10. Dvorackova E., Snoblova M., Hrdlicka P. // Int. Food Res. J. 2014. Vol. 21. No 4. pp. 1495-1500.

11. Koseoglu Yilmaz P., Kolak U. // Anal. Lett. 2016. Vol. 49. No 14. pp. 2157-2164.

12. Zhang Y., Shi P., Qu H., Cheng Y. // Rapid Commun. Mass Spectrom. 2007. Vol. 21. pp. 2971-2984.

13. Zgorka G., Kawka S. // J. Pharm. Biomed. Anal. 2001. Vol. 24. pp. 1065-1072.

14. Shutz K., Kammere D., Carle R., Schieber A. // J. Agric. Food Chem. 2004. Vol. 52. pp. 4090-4096.

15. Koseoglu Yilmaz P., Kolak U. // J. Chromatogr. Sci. 2017. Vol. 55. pp. 1-7.

16. Bielicka-Daszkiewicz, K. // Talanta. 2009. Vol. 80. pp. 614-621.

17. Magiera S., Zareba M. // Food Anal. Methods. 2015. Vol. 8. No 10. pp. 2665-2674.

18. Mothibedi K., Mokgadi J., Torto N. Agilent Technologies., Inc., 2011.

19. Simplified Solid Phase Extraction Solutions. Режим доступа: https://www.phenomenex.com (дата обращения: 28.11.2018). 
20. Милевская, В.В., Статкус М.А., Темердашев 3.А., Киселева Н.В. и др. // Журнал аналитической химии. 2016. Т. 71. № 7. С. 768-774.

21. Шилько Е.А., Милевская В.В., Темердашев 3.А., Киселева Н.В. // Аналитика $u$ контроль. 2018. Т. 22. № 3. С.303-314.

22. Ajila C., Brar S., Verma M., Tyagi R. et al. // Crit. Rev. Biotechnol. 2011. Vol. 31. No 3. pp. 227-249.

\section{References}

1. Zgorka G., Hajnos A., Chromatographia., 2003, Vol. 57, No 1, pp. 77-80. doi: 10.1007/BF02492087

2. Kozyra M., Glowniak K., Acta Soc. Bot. Pol., 2013, Vol. 82, No 4, pp. 325-329. doi: 10.5586/asbp.2013.039

3. Zeng H., Liu Z., Zhao S., Shu Y. et al., J. Sep. Sci., 2016, Vol. 39, No 19, pp. 3806-3817. doi: 10.1002/jssc.201600547

4. Glowniak K., Zgorka G., Kozyra M., J. Chromatogr. A., 1996, Vol. 730, pp. 25-29. doi: 10.1016/0021-9673(95)00877-2

5. Oniszczuk A., Podgorski R., Oniszczuk T., Zukiewicz-Sobczak W. et al., Industrial Crops and Products, 2014, Vol. 61, pp. 377-381. doi: 10.1016/j.indcrop.2014.07.036

6. Skrzypczak-Pietraszek E., Pietraszek J., J. Pharm. Biomed. Anal., 2012, Vol. 66, pp. 154161. doi: 10.1016/j.jpba.2012.03.037

7. Stephen Inbaraj B., Lu H., Kao T.H., Chen B.H., J. Pharm. Biomed. Anal., 2010, Vol. 51, pp. 549-556. doi: 10.1016/j.jpba.2009.09.006

8. Irakli M.N., Samanidou V.F., Biliaderis C.G., Papadoyannis I.N., J. Sep. Sci., 2012, Vol. 35, pp. 1603-1611. doi: 10.1002/jssc. 201200140

9. Ziakova A., Brandsteterova E., J. Liq. Chromatogr. Relat. Technol., 2002, Vol. 25, No 19, pp. 3017-3032. doi: 10.1081/JLC120015888

10. Dvorackova E., Snoblova M., Hrdlicka P., Int. Food Res. J., 2014, Vol. 21, No 4, pp.14951500.

11. Koseoglu Yilmaz P., Kolak U., Anal. Lett., 2016, Vol. 49, No 14, pp. 2157-2164. doi: 10.1080/00032719.2016.1145231

12. Zhang Y., Shi P., Qu H., Cheng Y., Rapid Commun. Mass Spectrom., 2007, Vol. 21, pp. 971-2984. doi: 10.1002/rcm.3166

13. Zgorka G., Kawka S., J. Pharm. Biomed. Anal., 2001, Vol. 24, pp. 1065-1072.
23. Fontanals N., Marce R., Borrull F. // Contributions to science. 2010. Vol. 6. No 2. pp. 199-213.

24. Buszewski B., Szultka M. // Crit. Rev. Anal. Chem.. 2012. Vol. 42. pp. 198-213.

25. Augusto F., Hantao L., Mogollon N., Braga S. // Trends Anal. Chem. 2012. Vol. 43. pp. 4-23.

26. Qureshi M., Stecher G., Huck C., Bonn G. // Cent. Eur. J. Chem.. 2011. Vol. 9. No 2. pp. 206-212.

14. Shutz K., Kammere D., Carle R., Schieber A., J. Agric. Food Chem., 2004, Vol. 52, pp. 4090-4096. doi: 10.1021/jf049625x

15. Koseoglu Yilmaz P., Kolak U., J. Chromatogr. Sci., 2017, Vol. 55, pp. 1-7. doi: 10.1093/chromsci/bmx025

16. Bielicka-Daszkiewicz, K., Talanta, 2009, Vol. 80, pp. 614-621. doi: 10.1016/j.talanta.2009.07.037

17. Magiera S., Zareba M., Food Anal. Methods. 2015, Vol. 8, No 10, pp. 2665-2674. doi: 10.1007/s12161-015-0166-y

18. Mothibedi K., Mokgadi J., Torto N., Agilent Technologies., Inc., 2011.

19. Simplified Solid Phase Extraction Solutions. Available at: https://www.phenomenex.com (accessed 28 November 2018).

20. Milevskaya V.V., Statkus M.A., Temerdashev Z. A, Kiseleva N.V. et al., Journal of Analytical Chemistry, 2016, 2016, Vol. 71, No.7, pp. 741-747. doi: 10.1134/S1061934816070133

21. Shil'ko E.A., Milevskaya V.V., Temerdashev Z. A, Kiseleva N.V., Analitika i kontrol'. 2018, Vol. 22, No 3, pp. 303-314. doi: 10.15826/analitika.2018.22.3.013

22. Ajila C., Brar S., Verma M., Tyagi R. et al., J., Crit. Rev. Biotechnol., 2011, Vol. 31, No 3, pp.227-249. doi: 10.3109/07388551.2010.513677

23. Fontanals N., Marce R., Borrull F., Contributions to science, 2010, Vol. 6, No 2, pp. 199-213. doi: 10.2436/20.7010.01.97

24. Buszewski B., Szultka M., Crit. Rev. Anal. Chem., 2012, Vol.42, pp. 198-213. doi: 10.1080/07373937.2011.645413

25. Augusto F., Hantao L., Mogollon N., Braga S., Trends Anal. Chem., 2012, Vol. 43, pp. 14-23. doi: 10.1016/j.trac.2012.08.012 
26. Qureshi M., Stecher G., Huck C., Bonn G., 206-212. doi: 10.2478/s11532-011-0006-x Cent. Eur. J. Chem., 2011, Vol. 9, No 2, pp.

Темердашев Зауаль Ахлоович - д.х.н., профессор, зав. кафедрой аналитической химии факультета химии и высоких технологий, Кубанский государственный университет, Краснодар.

Милевская Виктория Васильевна - к.Х.н., ст. преподаватель кафедры аналитической химии факультета химии и высоких технологий, Кубанский государственный университет, Краснодар.

Киселева Наталия Владимировна - к.х.н., доцент кафедры аналитической химии факультета химии и высоких технологий, Кубанский государственный университет, Краснодар.

Шилько Елена Александровна - аспирант кафедры аналитической химии факультета химии и высоких технологий, Кубанский государственный университет, Краснодар.
Temerdashev Zaual A. - doctor of chemical sciences, professor and the Head Department of Analytical Chemistry, Faculty of Chemistry and High Technologies, Kuban State University, Krasnodar. e-mail: temza@kubsu.ru

Milevskaya Victoria V. - Ph.D. in Chemistry, Art. Lecturer Department of Analytical Chemistry, Faculty of Chemistry and High Technologies, Kuban State University, Krasnodar. e-mail: milevskaya_victoriya@mail.ru

Kiseleva Natalia V. - Ph.D. in Chemistry, Associate Professor Department of Analytical Chemistry, Faculty of Chemistry and High Technologies, Kuban State University, Krasnodar. e-mail: lab284b@mail.ru

Shil'ko Elena A. - Ph. D. student of the Department of Analytical Chemistry, Faculty of Chemistry and High Technologies, Kuban State University, Krasnodar. e-mail: elenashilko94@gmail.com 